Journal of Engineering and Applied Sciences 14 (20): 7566-7573, 2019

ISSN: 1816-949X

(C) Medwell Journals, 2019

\title{
Study of New Mixture Distribution
}

\author{
${ }^{1}$ Maysaa Jalil Mohammed and ${ }^{2}$ Iden Hasan Hussein \\ ${ }^{1}$ Department of Mathematics, College of Education for Pure Sciences (Ibn Al-Haitham), \\ University of Baghdad, Baghdad, Iraq \\ ${ }^{2}$ Department of Mathematics, College of Sciences for Women, \\ University of Baghdad, Baghdad, Iraq
}

\begin{abstract}
This research is present the structure of a new mixture distribution by incorporating three distributions which have one parameter by utilizing the cumulative function of exponential pdf, standard Weibull pdf and Rayliegh pdf which is defined by "New Mixture Distribution". The method of mixing contains two main parts: The first part includes the process of mixing the distribution of exponential and rayliegh depending on the tail in each of them as well. The second part mixing the exponential and Weibull depending on the tail in each of them as well. Here, the exponential plays a big role in the mixing process in both parts. Based on the results of the first and second parts of the blending process, the new distribution was obtained. Moreover, realizing the mathematical and statistical properties of it. Furthermore, explaining the shapes of the density function and hazard rate function. Also, our investigation of $\mathrm{r}^{\text {th }}$ moment, incomplete moment, the moment generating function and characteristic function. Finally, obtaining the quantile function and any other statistical properties.
\end{abstract}

Key words: Exponential distribution, Rayleigh distribution, standard Weibull distribution, moments, quantile function, characteristic function

\section{INTRODUCTION}

Statistical applications play an important role in our life, especially in medicine and engineering which they have lifetime distributions such as Rayleigh, Weibull and exponential distributions. Waloddi Weibull invented the Weibull distribution in 1937 and delivered his hallmark American study on this subject in 1951. He claimed that his distribution applied to a wide range of problems which introduce by Nadarajah and Kotz (2005).

Lord Rayleigh (1880) introduced the Rayleigh distribution in connection with a problem in the field of acoustics. Since, then extensive work has taken place related to this distribution in different areas of science and technology. The Rayleigh distribution is a special case of the Weibull distribution with shape parameter equal to two. Which represents by Merovci and Elbatal (2015a, b). Also, the exponential distribution is a special case of Weibull distribution when the shape parameter of Weibull distribution is equal to one which represents by Merovci and Elbatal $(2015 \mathrm{a}, \mathrm{b})$. Working on finding new distributions derived from a particular distribution encouraged researchers to explore certain methods that would help them to mix distributions and form new distributions that may be more useful than the distributions generated in the statistical field, especially in the area of reliability and survival. Cordeiro et al. (2014) represents the new method of mixed the distributions depend on the tail of the distributions in 2011 and 2013. Nasiru (2016) introduce the new distribution depends on the tail of Weibull two parameters and the Rayleigh one parameter distributions. The new distribution is very flexible and can be used effectively in modeling survival and reliability problems. The aim of this study is to present the new mixture distribution called "New Mixture Distribution". This new distribution is dependent on the tail of mixed between exponential Rayleigh and exponential Weibull. The organization of this study includes the mathematical structure of this new distribution. Also, define the probability density function, cumulative density function, survival function, hazard function. Moreover, discuss the shapes of the density function and hazard rate function. Also, introduce the statistical properties of the new distribution. Finally, the conclusion of the study is determined.

Corresponding Author: Maysaa Jalil Mohammed, Department of Mathematics, College of Education for Pure Sciences (Ibn Al-Haitham), University of Baghdad, Baghdad, Iraq 


\section{MATERIALS AND METHODS}

The mathematical structure of this new distribution takes three parts. First part, mixed between exponential and standard Weibull distributions both have one parameter as follows: the pdf's functions are:

$$
f_{\text {Exp }}(y)=\Upsilon^{-Y_{y}} \text { and } f_{\text {Weab }}(z)=\alpha z^{\alpha-1} e^{-z^{\alpha}}
$$

The cdf's functions are as follows:

$$
F_{\text {Exp }}(y)=1-e^{-Y Y} \text { and } F_{W e i b}(z)=1-e^{-z^{\alpha}}
$$

The generalized exponential standard Weibull distribution with cdf function is:

$$
F_{E W}(t)=1-e^{-\left(r t+t^{\alpha}\right)}
$$

where, $\mathrm{T}=\min \{\mathrm{Y}, \mathrm{Z}\}, \mathrm{Y}$ and $\mathrm{Z}$ are two independent random variables. Second part, mixed between exponential and Rayleigh distributions both have one parameter as follows: the pdf's functions are as follows:

$$
f_{\text {Exp }}(v)=\Upsilon^{-Y_{v}} \text { and } f_{\text {Ray }}(u)=\beta e^{-\frac{\beta}{2} u^{2}}
$$

The cdf's functions are as follows:

$$
F_{\text {Exp }}(v)=1-e^{-Y v} \text { and } F_{\text {Ray }}(u)=1-e^{-\frac{\beta}{2} u^{2}}
$$

The generalized exponential Rayleigh distribution with cdf function is:

$$
F_{E R}(k)=1-e^{-\left(Y k+\frac{B}{2} k^{2}\right)}
$$

where, $\mathrm{k}=\mathrm{MIN}\{\mathrm{V}, \mathrm{U}\}, \mathrm{V}$ and $\mathrm{U}$ are two independent random variables. Third part using the result from Eq. 1 and 2 to mix between them the final result is the new mixture distribution with cdf function:

$$
F(x)=1-e^{-\left(2 \gamma x+\frac{\beta}{2} x^{2}+x^{\alpha}\right)}, x>0
$$

where, $X=\min \{K, T\}, \Upsilon>0, \beta>0$ are two scale parameters and $\alpha>0$ is the shape parameter of a new mixture distribution and pdf function form is:

$$
f(x)=\left(2 \Upsilon+\beta x+\alpha x^{(\alpha-1)}\right) e^{-\left(2 \Upsilon x+\frac{\beta}{2} x^{2}+x^{\alpha}\right)}, x>0
$$

It is observed that, the proposed new mixture distribution has several interesting properties and it can used effectively to analyze them. The survival function of new mixture distribution is:

$$
S(x)=e^{-\left(2 Y x+\frac{\beta}{2} x^{2}+z^{\alpha}\right)}, x>0
$$

The hazard rate function is:

$$
h(x)=2 \Upsilon+\beta x+\alpha x^{\alpha-1}, x>0
$$

It is clear that, if $\Upsilon=0$, the new mixture pdf transform to Weibull-Rayliegh pdf, if $\beta=0$, the new mixture pdf transform to exponential-Weibull pdf. Furthermore, if $\alpha \neq 0$, $\beta=0$ and $\Upsilon=0$ then, the new mixture distribution reduced to standard Weibull distribution (Fig. 1 and 2).

Shapes: Discuss the shapes of the density and of the hazard rate functions. Consider:

$$
\lim _{x \rightarrow 0} f(x)= \begin{cases}\infty & \alpha<1 \\ 2 \Upsilon & \alpha>1\end{cases}
$$

and $\lim _{x \rightarrow \infty} f(x)=0$. Then:

$$
\begin{aligned}
& \ln f(x)=\ln \left(2 \Upsilon+\beta x+\alpha x^{\alpha-1}\right)-\left(2 \Upsilon x+\frac{\beta}{2} x^{2}+x^{\alpha}\right) \\
& \frac{\partial \ln f(x)}{\partial x}=\frac{\beta+\alpha(\alpha-1) x^{\alpha-2}}{2 \Upsilon+\beta x+\alpha x^{\alpha-1}}-\left(2 \Upsilon+\beta x+\alpha x^{\alpha-1}\right)=0
\end{aligned}
$$

Then:

$$
\beta+\alpha(\alpha-)(\alpha-1) x^{\alpha-2}=\left(2 \Upsilon+\beta x+\alpha x^{(\alpha-1)}\right)^{2}
$$

There are more than one root of this equation. So, if $\mathrm{x}=\mathrm{x}_{1}$ is the root of the equation, then, it depending to a local maximum, minimum or a point of inflection which depending on the $\mathrm{J}\left(\mathrm{x}_{1}\right)<0, \mathrm{~J}\left(\mathrm{x}_{1}\right)>0$ or $\mathrm{J}\left(\mathrm{x}_{1}\right)=0$. Where:

$$
\begin{aligned}
& \frac{\partial \operatorname{lnf}(x)}{\partial x}=\left(\left(\beta+\alpha(\alpha-1) x^{\alpha-2}\right)\left(2 \Upsilon+\beta x+\alpha x^{\alpha-1}\right)^{-1}-\right. \\
& \left(2 \Upsilon+\beta x+\alpha x^{\alpha-1}\right) J(x)=\frac{\partial^{2} \ln f(x)}{\partial x^{2}}=- \\
& \left(\beta+\alpha(\alpha-1) x^{\alpha-2}\right)^{2}+\left(2 \Upsilon+\beta x+\alpha x^{\alpha-1}\right)^{-2} \\
& +\left(2 \Upsilon+\beta x+\alpha x^{\alpha-1}\right)^{-1}\left(\alpha(\alpha-1)(\alpha-2) x^{\alpha-3}\right)- \\
& \left(\beta+\alpha(\alpha-1) x^{\alpha-2}\right)
\end{aligned}
$$

Moreover, since, the hazard rate function is: $h(x)=2 \Upsilon+\beta x+\alpha x^{\alpha-1}$ :

$$
h^{\prime}(x)=\beta+a(a-1) x^{a-2}, \quad x>0
$$


J. Eng. Applied Sci., 14 (20): 7566-7573, 2019

(a)

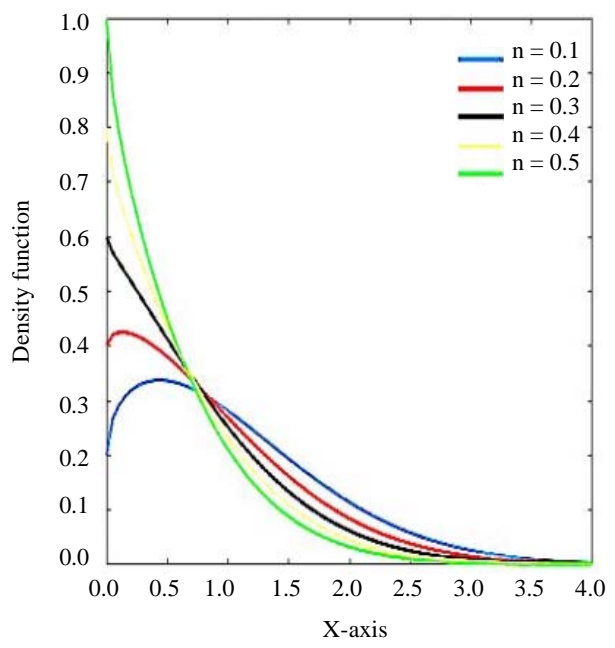

(c)

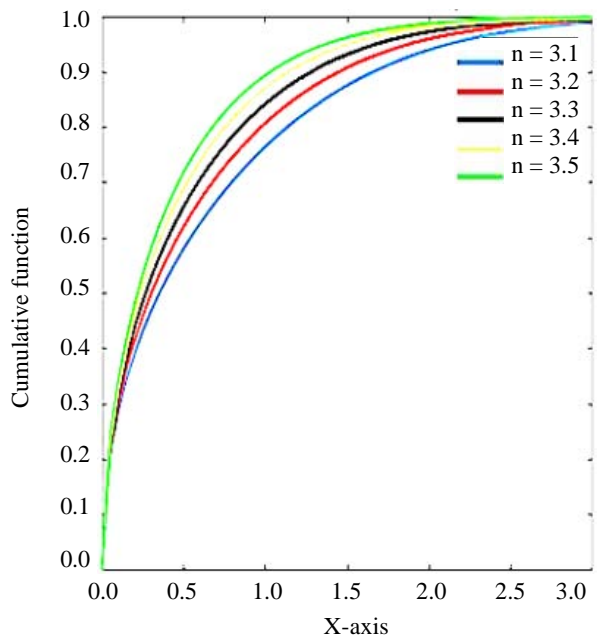

(e)

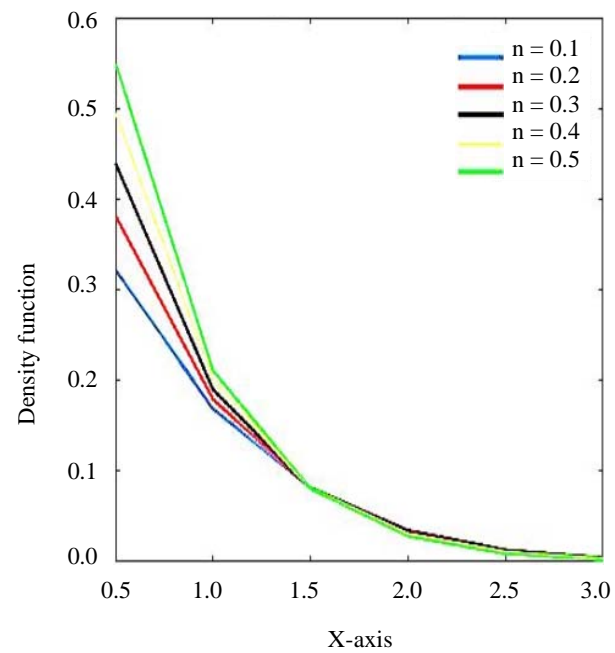

(b)

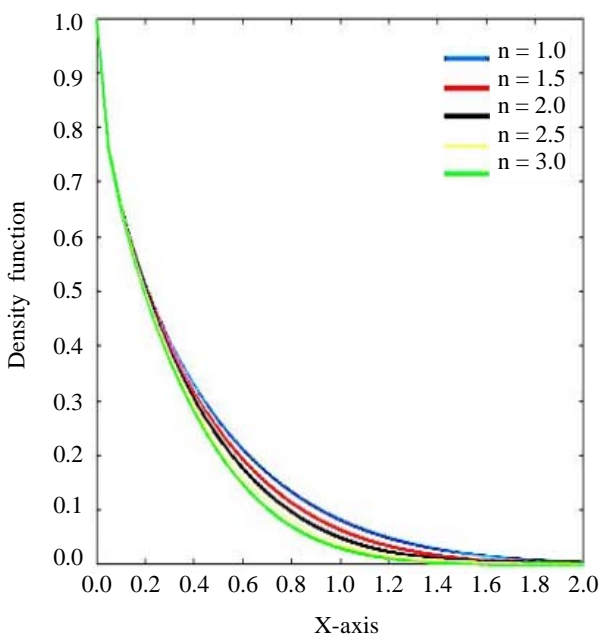

(d)

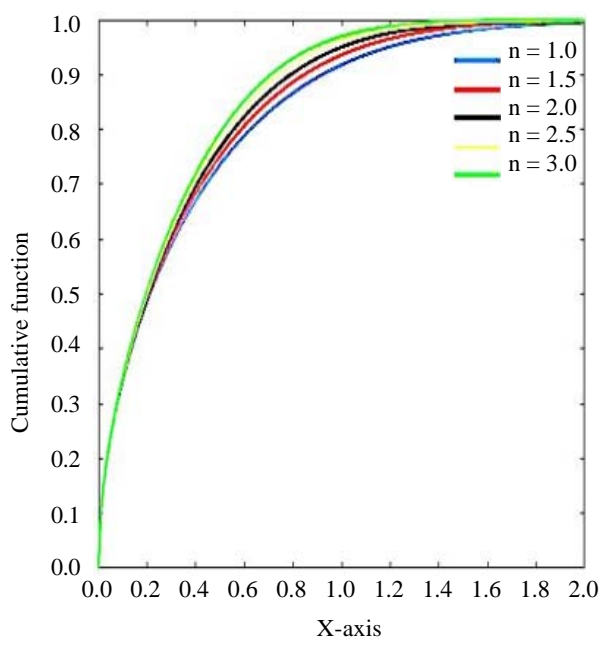

(f)

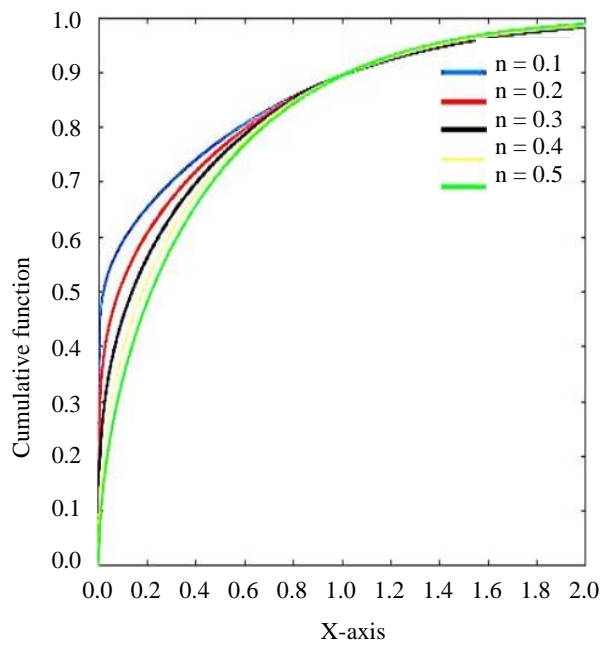

Fig. 1: The density and cumulative functions of new mixture distribution 

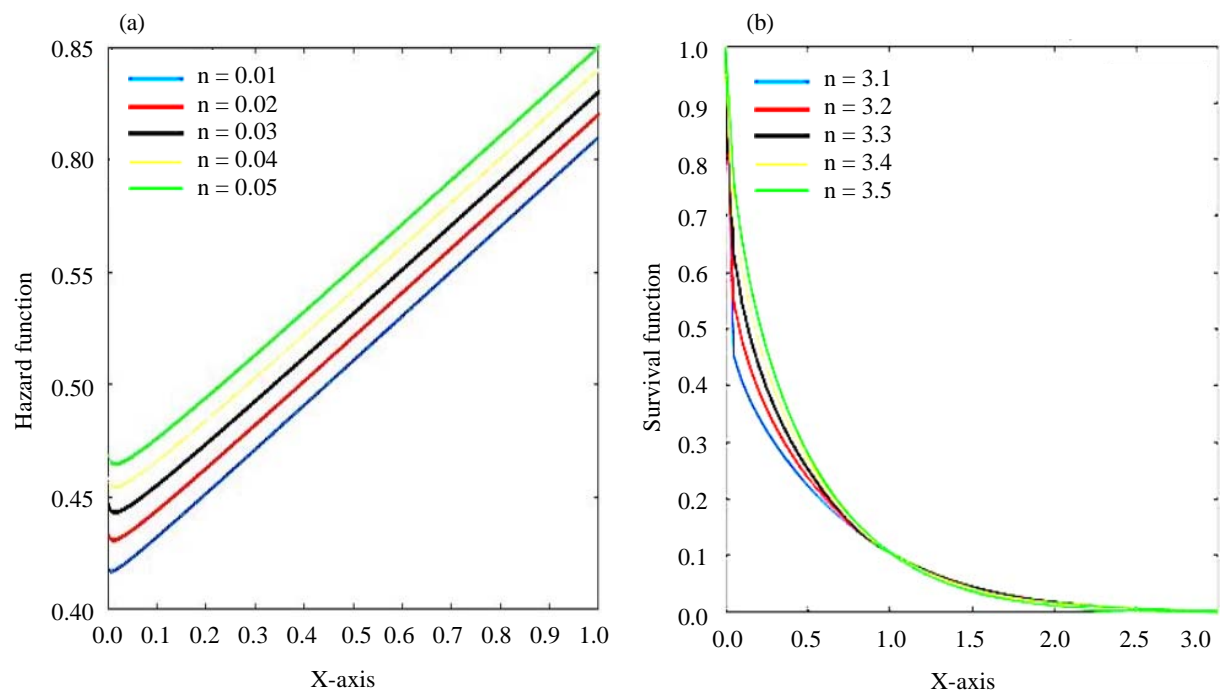

Fig. 2: Shape of survival and hazard rate functions of new mixture distribution

The shape of hazard function depends on the parameter $(\alpha)$ such that Xie and Lai (1996). If $\alpha<1$, then, $h(x)$ will be decreasing function that is $x>0$ and $h^{\prime}(x)<0$. If $\alpha>1$, then, $\mathrm{h}(\mathrm{x})$ will be increasing function that is $\mathrm{x}>0$ and $h^{\prime}(x)>0$ :

$$
\lim _{x \rightarrow 0} h(x)= \begin{cases}\infty & \alpha<1 \\ 2 \Upsilon & \alpha<1\end{cases}
$$

And:

$$
\lim _{x \rightarrow 0} h(x)= \begin{cases}2 \Upsilon & \alpha<1 \\ \infty & \alpha<1\end{cases}
$$

\section{RESULTS AND DISCUSSION}

New mixture distribution properties: In the following, the discussion of statistical properties of new mixture distribution:

\section{Moments}

Theorem 1: The rth moment of new mixture distribution is:

$$
\begin{aligned}
& \mathrm{M}_{\mathrm{r}}^{\prime}=\mathrm{E}\left(\mathrm{x}^{\mathrm{r}}\right)=2 \Upsilon \mathrm{YK}(\mathrm{r}, \Upsilon, \beta, \alpha)+ \\
& \beta \mathrm{K}(\mathrm{r}+1, \Upsilon, \beta, \alpha)+\alpha \mathrm{K}(\mathrm{r}+\alpha-1, \Upsilon, \beta, \alpha)
\end{aligned}
$$

\section{Proof:}

$$
\begin{aligned}
& E\left(x^{r}\right)=\int_{0}^{\infty} x^{r} f(x) d x=\int_{0}^{\infty} x^{r}\left(2 \Upsilon+\beta x+\alpha x^{\alpha-1}\right) e^{-\left(2 r x+\frac{\beta}{2} x^{2}+z^{\alpha}\right)} d x \\
& \text { Let } K(r, \Upsilon, \beta, \alpha)=\int_{0}^{\infty} x^{r} e^{-\left(2 r x+\frac{\beta}{2} z^{2}+z^{\alpha}\right)} d x
\end{aligned}
$$

Expanding $\left(\mathrm{e}^{-2 \mathrm{Y}}\right)$ and $\left(\mathrm{e}-\beta / 2 \mathrm{x}^{2}\right)$ as Taylor series as follows:

$$
K(r, \Upsilon, \beta, \alpha)=\sum_{n=0}^{\infty} \sum_{m=0}^{\infty} \frac{(-2 \Upsilon)^{n}}{n !} \frac{(-\beta)^{m}}{2^{m} m !} \int_{0}^{\infty} x^{r+n+2 m} e^{-z^{\alpha}} d x
$$

Then:

$$
\int_{0}^{\infty} x^{x+n+2 m} e^{-x^{2}} d x
$$

Let:

$$
\begin{aligned}
& u=x^{r+n+2 m+1}, d u=(r+n+2 m+1) x^{r+n+2 m} d x \\
& d x=\frac{d u}{(r+n+2 m+1) x^{r+n+2 m}}(\#) \\
& u=x^{r+n+2 m+1} \text {, then, } u^{\frac{\alpha}{r+n+2 m+1}}=x^{\alpha}
\end{aligned}
$$

From (\#) and (\#\#) get:

$$
\begin{aligned}
& \int_{0}^{\infty} \mathrm{x}^{\mathrm{r}+\mathrm{n}+2 \mathrm{~m}} \mathrm{e}^{-\mathrm{x}^{\alpha}} \mathrm{dx}=\int_{0}^{\infty} \mathrm{x}^{\mathrm{r}+\mathrm{n}+2 \mathrm{~m}} \mathrm{e}^{-\mathrm{u}^{\frac{\alpha}{x+n+2 n}}} \frac{\mathrm{du}}{(\mathrm{r}+\mathrm{n}+2 \mathrm{~m}+1) \mathrm{x}^{\mathrm{x}^{\mathrm{r}+\mathrm{n}+2 \mathrm{~m}}}} \\
& =\frac{1}{\mathrm{r}+\mathrm{n}+2 \mathrm{~m}+1} \int_{0}^{\infty} \mathrm{e}^{-\mathrm{u}^{\frac{\alpha}{\mathrm{r}+\mathrm{n}+2 m}}} \mathrm{du}=\frac{1}{\mathrm{r}+\mathrm{n}+2 \mathrm{~m}+1} \\
& \frac{-(\mathrm{r}+\mathrm{n}+2 \mathrm{~m}+1) \Gamma\left(\frac{\mathrm{r}+\mathrm{n}+2 \mathrm{~m}+1}{\alpha}, \mathrm{u}^{\frac{\alpha}{\mathrm{r}+\mathrm{n}+2 \mathrm{~m}+1}}\right)}{\mathrm{a}}= \\
& \frac{-\Gamma\left(\frac{\mathrm{r}+\mathrm{n}+2 \mathrm{~m}+1}{\alpha}, \mathrm{x}^{\alpha}\right)}{\alpha}
\end{aligned}
$$

This is a special integral (incomplete Gamma function) 


$$
\begin{aligned}
& \mathrm{K}(\mathrm{r}, \Upsilon, \beta, \alpha)=\frac{1}{\alpha} \sum_{\mathrm{n}=0}^{\infty} \sum_{\mathrm{m}=0}^{\infty} \frac{(-2 Y)^{\mathrm{n}}}{\mathrm{n} !} \frac{(-\beta)^{\mathrm{m}}}{2^{\mathrm{m}} \mathrm{m} !} \mathrm{G}\left(\frac{\mathrm{r}+\mathrm{n}+2 \mathrm{~m}+1}{\alpha}\right) \\
& \mathrm{K}(\mathrm{r}, \Upsilon, \beta, \alpha)=\frac{1}{\alpha}{ }_{i}{ }_{0}\left[\left(\frac{\mathrm{r}+1}{\alpha}, \frac{1}{\alpha}\right),-\alpha\right]
\end{aligned}
$$

where, ${ }_{1} \psi_{0}$ is the Wright generalized hypergeometric function which is defined in the appendix by Wright (1935). Then:

$$
\begin{aligned}
& M_{r}^{\prime}=E\left(x^{r}\right)= \\
& 2 \Upsilon K(r, \Upsilon, \beta, \alpha)+ \\
& \beta K(r+1, \Upsilon, \beta, \alpha)+\alpha K(r+\alpha-1, \Upsilon, \beta, \alpha)
\end{aligned}
$$

Where:

$$
\begin{aligned}
& \mathrm{K}(\mathrm{r}, \Upsilon, \beta, \alpha)=\frac{1}{\alpha}{ }_{1} ?_{0}\left[\left(\frac{\mathrm{r}+1}{\alpha}, \frac{1}{\alpha}\right),-\alpha\right] \\
& \mathrm{K}(\mathrm{r}+1, \Upsilon, \beta, \alpha)=\frac{1}{\alpha}{ }_{1} ?_{0}\left[\left(\frac{\mathrm{r}+2}{\alpha}, \frac{1}{\alpha}\right),-\alpha\right] \\
& \mathrm{K}(\mathrm{r}+\alpha-1, \Upsilon, \beta, \alpha)=\frac{1}{\alpha}{ }_{1} ?_{0}\left[\left(\frac{\mathrm{r}+\alpha}{\alpha}, \frac{1}{\alpha}\right),-\alpha\right]
\end{aligned}
$$

Then, the mean of the new mixture distribution is:

$$
\begin{aligned}
& \mathrm{E}(\mathrm{x})=2 \Upsilon \mathrm{K}(1, \Upsilon, \beta, \alpha)+ \\
& \beta \mathrm{K}(2, \Upsilon, \beta, \alpha)+\alpha \mathrm{K}(\alpha, \Upsilon, \beta, \alpha)
\end{aligned}
$$

And, the second moment of the new mixture distribution is:

$$
\begin{aligned}
& \mathrm{E}\left(\mathrm{x}^{2}\right)=2 \Upsilon \mathrm{K}(2, \Upsilon, \beta, \alpha)+ \\
& \beta \mathrm{K}(3, \Upsilon, \beta, \alpha)+\alpha \mathrm{K}(\alpha+1, \Upsilon, \beta, \alpha)
\end{aligned}
$$

Then, the variance of the new mixture distribution is:

$$
\begin{aligned}
& \operatorname{Var}(\mathrm{x})=\mathrm{E}\left(\mathrm{x}^{2}\right)+[\mathrm{E}(\mathrm{x})]^{2}= \\
& 2 \Upsilon \mathrm{K}(2, \Upsilon, \beta, \alpha)+\beta \mathrm{K}(3, \Upsilon, \beta, \alpha)+ \\
& \alpha \mathrm{K}(\alpha+1, \Upsilon, \beta, \alpha)+ \\
& {[2 \Upsilon \mathrm{YK}(1, \Upsilon, \beta, \alpha)+\beta \mathrm{K}(2, \Upsilon, \beta, \alpha)+\alpha \mathrm{K}(\alpha, \Upsilon, \beta, \alpha)]^{2}}
\end{aligned}
$$

Incomplete moments: In life time model, one of the big important things is to know the $\mathrm{r}^{\text {th }}$ incomplete moments which is given by $T_{r}(y)=E\left(X^{r} \mid X \leq y\right)$. For getting the rth incomplete moments define:

$$
I(r, a)=I(r, a, \beta, \alpha)=\int_{0}^{\infty} x^{r} e^{-z^{\alpha}} d x
$$

From Eq. 2, rewrite $\mathrm{T}_{\mathrm{r}}(\mathrm{y})=\int_{0}^{\infty} \mathrm{x}^{\mathrm{x} f} \mathrm{f}(\mathrm{x}) \mathrm{dx}$ and it's possible to represent as:

$$
\begin{aligned}
& T_{r}(y)=\int_{0}^{y} x^{r}\left(2 \Upsilon+\beta x+\alpha x^{\alpha-1}\right) e^{\left(2 \gamma x+\frac{\beta}{2} z^{2}+z^{\alpha}\right)} d x \\
& \mathrm{~T}_{\mathrm{r}}(\mathrm{y})=\sum_{\mathrm{n}=0}^{\infty} \sum_{\mathrm{m}=0}^{\infty} \frac{(-2 \Upsilon)^{\mathrm{n}}}{\mathrm{n} !} \frac{(-\beta)^{\mathrm{m}}}{2^{\mathrm{m}} \mathrm{m} !} \int_{0}^{\mathrm{y}} \mathrm{x}^{\mathrm{x}+\mathrm{n}+2 \mathrm{~m}} \mathrm{e}^{-\mathrm{x}^{\alpha}} \mathrm{dx} \\
& \mathrm{T}_{\mathrm{r}}(\mathrm{y})=\sum_{\mathrm{n}=0 \mathrm{~m}=0}^{\infty} \sum_{[2 \Upsilon !}^{\infty} \frac{(-2 \Upsilon)^{\mathrm{n}}}{\mathrm{n} !} \frac{(-\beta)^{\mathrm{m}}}{2^{\mathrm{m}} \mathrm{m} !}
\end{aligned}
$$

Where:

$$
I(r+n+2 m, y)=\int_{0}^{y} x^{r+n+2 m} e^{-z^{\alpha}} d x
$$

By using the Meijer function which is define in the appendix can find the value of integration of Eq. 8 by Kiryakova (2002) as follows:

$$
\exp (-\mathrm{g}(\mathrm{x}))=\mathrm{G}_{0,1}^{1,0}\left(\mathrm{~g}(\mathrm{x}) \mathrm{I}_{0}\right)
$$

From Eq. 8:

$$
I(r, a)=\int_{0}^{y} x^{r} G_{0,1}^{1,0}\left(\left.x^{s / j}\right|_{0} ^{j}\right) d x
$$

By Prudnikov et al. (1986) using equation (2.24.2.2), which is define in the appendix can represent as $\mathrm{I}(\mathrm{r}, \mathrm{a})$ where:

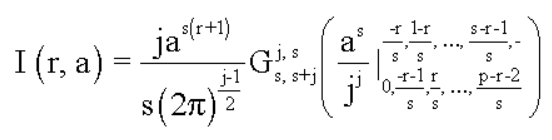

where, $\alpha=s / j$ where, $s$ and $j$ are co-prime numbers as represents by Jeffrey and Zwillinger (2007). Compering Eq. 9 and 11 with the equation (2.24.2.2) satisfy the equation of incomplete moment. Here:

$$
\begin{aligned}
& \beta=1, \alpha-1=r \text { then } r+1=\alpha, \\
& m=1, n=0, s=0, j=1, c=j, 1=s \\
& \mu=1, w^{*}=\frac{1}{2} \text { and } \omega=1
\end{aligned}
$$

\section{Moments generating function}

Theorem 2: The (mgf) of $\mathrm{X}, \mathrm{M}(\mathrm{t})=\mathrm{E}\left(\mathrm{e}^{\mathrm{tx}}\right)$ is given by $\mathrm{M}(\mathrm{t})=2 \mathrm{YK}(\mathrm{t}, 2 \mathrm{Y}-\mathrm{t}, \beta, \alpha)+\beta \mathrm{K}(\mathrm{t}+1,2 \mathrm{Y}-\mathrm{t}, \beta, \alpha)+\alpha \mathrm{K}(\mathrm{t}+\alpha-1$, $2 \mathrm{Y}-\mathrm{t}, \beta, \alpha)$ :

\section{Proof:}




$$
\begin{aligned}
& M(t)=\int_{0}^{\infty} E\left(e^{t x}\right)=\int_{0}^{\infty} e^{t \mathrm{tx}} f(x) d x= \\
& \int_{0}^{\infty} \mathrm{e}^{\mathrm{tz}}\left(2 \Upsilon+\beta \mathrm{x}+\alpha \mathrm{x}^{\alpha-1}\right) \mathrm{e}^{-\left(2 \Upsilon \mathrm{x}+\frac{\beta}{2} \mathrm{x}^{2}+\mathrm{z}^{\alpha}\right)} \mathrm{dx} \\
& \int_{0}^{\infty}\left(2 \Upsilon+\beta \mathrm{x}+\alpha \mathrm{x}^{\alpha-1}\right) \mathrm{e}^{-\left[(2 \Upsilon-\mathrm{t}) \mathrm{x}+\frac{\beta}{2} \mathrm{x}^{2}+\mathrm{z}^{\alpha}\right]} \mathrm{dx} \\
& \text { Let } \mathrm{K}(\mathrm{t}, 2 \Upsilon-\mathrm{t}, \beta, \alpha)=\int_{0}^{\infty} \mathrm{e}^{-\left[(2 \Upsilon-\mathrm{t}) \mathrm{x}+\frac{\beta}{2} \mathrm{x}^{2}+\mathrm{z}^{\alpha}\right]} \mathrm{dx} \\
& \mathrm{K}(\mathrm{t}, 2 \Upsilon-\mathrm{t}, \beta, \alpha)=\frac{1}{\alpha} \sum_{\mathrm{n}=0}^{\infty} \sum_{\mathrm{m}=0}^{\infty} \frac{(2 \Upsilon-\mathrm{t})^{\mathrm{n}}}{\mathrm{n} !} \frac{(-\beta)^{\mathrm{m}}}{2^{\mathrm{m}} \mathrm{m} !} \Gamma
\end{aligned}
$$

$\mathrm{M}(\mathrm{t})=2 \mathrm{YK}(\mathrm{t}, 2 \mathrm{Y}-\mathrm{t}, \beta, \alpha)+\beta \mathrm{K}(\mathrm{t}+1,2 \mathrm{Y}-\mathrm{t}, \beta, \alpha)+\alpha \mathrm{K}(\mathrm{t}+\alpha-1,2$ $\mathrm{Y}-\mathrm{t}, \beta, \alpha)$. Where:

$$
\begin{aligned}
& \mathrm{K}(\mathrm{t}, 2 \Upsilon-\mathrm{t}, \beta, \alpha)= \\
& \frac{1}{\alpha} \sum_{\mathrm{n}=0}^{\infty} \sum_{\mathrm{m}=0}^{\infty} \frac{(2 \Upsilon-\mathrm{t})^{\mathrm{n}}}{\mathrm{n} !} \frac{(-\beta)^{\mathrm{m}}}{2^{\mathrm{m}} \mathrm{m} !} \Gamma\left(\frac{\mathrm{t}+\mathrm{n}+2 \mathrm{~m}+2}{\alpha}\right) \\
& \mathrm{K}(\mathrm{t}+1,2 \Upsilon-\mathrm{t}, \beta, \alpha)= \\
& \frac{1}{\alpha} \sum_{\mathrm{n}=0}^{\infty} \sum_{\mathrm{m}=0}^{\infty} \frac{(2 \Upsilon-\mathrm{t})^{\mathrm{n}}}{\mathrm{n} !} \frac{(-\beta)^{\mathrm{m}}}{2^{\mathrm{m}} \mathrm{m} !} \Gamma\left(\frac{\mathrm{t}+\mathrm{n}+2 \mathrm{~m}+2}{\alpha}\right)
\end{aligned}
$$

And:

$$
\begin{aligned}
& \mathrm{K}(\mathrm{t}+1,2 \Upsilon-\mathrm{t}, \beta, \alpha)= \\
& \frac{1}{\alpha} \sum_{\mathrm{n}=0}^{\infty} \sum_{\mathrm{m}=0}^{\infty} \frac{(2 \Upsilon-\mathrm{t})^{\mathrm{n}}}{\mathrm{n} !} \frac{(-\beta)^{\mathrm{m}}}{2^{\mathrm{m}} \mathrm{m} !} \Gamma\left(\frac{\mathrm{t}+\mathrm{n}+2 \mathrm{~m}+2}{\alpha}\right)
\end{aligned}
$$

\section{Factorial moments generating function}

Theorem 3: The factorial moments generating function of $\mathrm{X}$ denoted by: $\mathrm{M}_{\mathrm{z}}(\mathrm{t})=2 \mathrm{YK}(\mathrm{t}, 2 \mathrm{Y}-\mathrm{In} \mathrm{t}, \beta, \alpha)+\beta \mathrm{K}(\mathrm{t}+1,2 \mathrm{Y}-\mathrm{In}$ $\mathrm{t}, \beta, \alpha)+\alpha \mathrm{K}(\mathrm{t}+\alpha-1,2 \mathrm{Y}-\mathrm{In} \mathrm{t}, \beta, \alpha)$.

\section{Proof:}

$$
\begin{aligned}
& M_{z}(t)=E\left(t^{x}\right)=\int_{0}^{\infty} t^{x} f(x) d x= \\
& \int_{0}^{\infty} e^{\ln t^{x}} f(x) d x=\int_{0}^{\infty} e^{x \ln t} f(x) d x= \\
& \int_{0}^{\infty} e^{x \ln t}\left(2 \Upsilon+\beta x+\alpha x^{\alpha-1}\right) e^{-\left(2 \Upsilon x+\frac{\beta}{2} x^{2}+x^{\alpha}\right)} d x= \\
& \int_{0}^{\infty}\left(2 \Upsilon+\beta x+\alpha x^{\alpha-1}\right) e^{-\left(2 Y x+\frac{\beta}{2} x^{2}+x^{\alpha}\right)} d x \\
& \left.\operatorname{let} K(t, 2 \Upsilon-\ln t, \beta, \alpha)=\int_{0}^{\infty} e^{-[(2 Y-\ln t))+\frac{\beta}{2} x^{2}+x^{\alpha}}\right] d x \\
& K(t, 2 \Upsilon-\ln t, \beta, \alpha)= \\
& \frac{1}{\alpha} \sum_{n=0}^{\infty} \sum_{m=0}^{\infty} \frac{(2 \Upsilon-\ln t))^{n}}{n !} \frac{(-\beta)^{m}}{2^{m} m !} \Gamma\left(\frac{t+n+2 m+1}{\alpha}\right)
\end{aligned}
$$

That yield, $\mathrm{M}(\mathrm{t})=2 \mathrm{YK}(\mathrm{t}, 2 \mathrm{Y}-\mathrm{In} \mathrm{t}, \beta, \alpha)+\beta \mathrm{K}(\mathrm{t}+1$, $2 \mathrm{Y}-\beta, \alpha)+\alpha \mathrm{K}(\mathrm{t}+1,2 \mathrm{Y}-\beta, \alpha)$. Where:

$\mathrm{K}(\mathrm{t}, 2 \Upsilon-\ln \mathrm{t}, \beta, \alpha)=$

$$
\begin{aligned}
& \frac{1}{\alpha} \sum_{n=0}^{\infty} \sum_{m=0}^{\infty} \frac{(2 \Upsilon-I n t))^{n}}{n !} \frac{(-\beta)^{m}}{2^{m} m !} \Gamma\left(\frac{t+n+2 m+1}{\alpha}\right) \\
& K(t+1,2 \Upsilon-\ln t, \beta, \alpha)= \\
& \frac{1}{\alpha} \sum_{n=0}^{\infty} \sum_{m=0}^{\infty} \frac{(2 \Upsilon-I n t))^{n}}{n !} \frac{(-\beta)^{m}}{2^{m} m !} \Gamma\left(\frac{t+n+2 m+1}{\alpha}\right)
\end{aligned}
$$

And:

$$
\begin{aligned}
& \mathrm{K}(\mathrm{t}+\alpha-1,2 \Upsilon-\ln \mathrm{t}, \beta, \alpha)= \\
& \frac{1}{\alpha} \sum_{\mathrm{n}=0}^{\infty} \sum_{\mathrm{m}=0}^{\infty} \frac{(2 \Upsilon-\mathrm{In} \mathrm{t}))^{\mathrm{n}}}{\mathrm{n} !} \frac{(-\beta)^{\mathrm{m}}}{2^{\mathrm{m}} \mathrm{m} !} \Gamma\left(\frac{\mathrm{t}+\mathrm{n}+2 \mathrm{~m}+1}{\alpha}\right)
\end{aligned}
$$

Quantile function: The quantile function is denoted by $\mathrm{x}=\mathrm{Q}(\mathrm{v})=\mathrm{F}^{-1}(\mathrm{v})$. Where:

$$
\begin{aligned}
& F(x)=1-e^{-\left(2 \Upsilon x+\frac{\beta}{2} x^{2}+z^{\alpha}\right)}, v=1-e^{-\left(2 \Upsilon x+\frac{\beta}{2} x^{2}+z^{\alpha}\right)} \\
& \text { and } 1-\mathrm{v}=\mathrm{e}^{-\left(2 \mathrm{Y}+\frac{\mathrm{B}}{2} \mathrm{x}^{2}+\mathrm{x}^{\alpha}\right)}
\end{aligned}
$$

This yield:

$$
\begin{aligned}
& \left(2 \Upsilon x+\frac{\beta}{2} x^{2}+x^{\alpha}\right)=-\ln (1-v) \\
& \text { let } z=\left(2 \Upsilon x+\frac{\beta}{2} x^{2}+x^{\alpha}\right)
\end{aligned}
$$

Depended on Taylor series represent:

$$
x^{\alpha}=\sum_{i=0}^{\infty} c_{i} x^{i} \text { where, } c_{i}=\sum_{n=0}^{\infty} \frac{(-1)^{n-1}\left(\begin{array}{l}
n \\
i
\end{array}\right)(\alpha)_{n}}{n !}
$$

And, $(\alpha)_{\mathrm{n}}=\alpha(\alpha-1), \ldots,(\alpha-\mathrm{n}+1)$ is the descending factorial. Let, $z=\sum_{i=0}^{\infty} \phi_{i} x^{i}$ such that:

$$
\begin{aligned}
& \phi_{\circ}=c_{o}, \phi_{1}=c_{1}+\frac{\beta}{2}, \phi_{2}=c_{2}+\frac{\beta}{2} c_{1}+2 \Upsilon \text { and } \\
& \phi_{i}=c_{i} \text { for } i \geq 3
\end{aligned}
$$

If $\mathrm{c}_{1} \neq 0$ reverse the last series and acquire (equation 25.2.60) represents by Abramowitz and Stegun (1970). $\mathrm{x}=\mathrm{Q}(\mathrm{v})=\sum_{\mathrm{i}=0}^{\infty} \mathrm{S} \mathrm{S}_{\mathrm{i}}^{\mathrm{i}}$ such that. Where: 
And so, on.

$$
\begin{aligned}
& \mathrm{s}_{1}=\phi_{1}^{-1}, \mathrm{~s}_{2}=-\phi_{2} \phi_{1}^{-3} \\
& \text { and } \mathrm{s}_{3}=\left(2 \phi_{2}{ }^{2}-\phi_{1} \phi_{3}\right) \phi_{1}^{-5}
\end{aligned}
$$

Skewness: The skewness of new mixture distribution is dependent on moments (Merovci and Elbata, 2015a, b) denoted by:

Where:

$$
\mathrm{C} . \mathrm{S}=\frac{\mathrm{M}_{3}}{\left(\mathrm{M}_{2}\right)^{\frac{3}{2}}}
$$

$$
\begin{aligned}
& \mathrm{M}_{3}=\mathrm{E}\left(\mathrm{x}^{3}\right)= \\
& 2 \Upsilon \mathrm{YK}(3, \Upsilon, \beta, \alpha)+4 \mathrm{~K}(3, \Upsilon, \beta, \alpha)+ \\
& \alpha \mathrm{K}(2, \Upsilon, \beta, \alpha) \\
& \text { and } \mathrm{K}(3, \Upsilon, \beta, \alpha)= \\
& \frac{1}{\alpha} \sum_{\mathrm{n}=0}^{\infty} \sum_{\mathrm{m}=0}^{\infty} \frac{(-2 \Upsilon)^{\mathrm{n}}}{\mathrm{n} !} \frac{(-\beta)^{\mathrm{m}}}{2^{\mathrm{m}} \mathrm{m!}} \Gamma\left(\frac{4+\mathrm{n}+2 \mathrm{~m}}{\alpha}\right) \\
& \mathrm{K}(4, \Upsilon, \beta, \alpha)= \\
& \frac{1}{\alpha} \sum_{\mathrm{n}=0}^{\infty} \sum_{\mathrm{m}=0}^{\infty} \frac{(-2 \Upsilon)^{\mathrm{n}}}{\mathrm{n} !} \frac{(-\beta)^{\mathrm{m}}}{2^{\mathrm{m}} \mathrm{m} !} \Gamma\left(\frac{5+\mathrm{n}+2 \mathrm{~m}}{\alpha}\right) \\
& \mathrm{K}(\alpha+2, \Upsilon, \beta, \alpha)= \\
& \frac{1}{\alpha} \sum_{\mathrm{n}=0}^{\infty} \sum_{\mathrm{m}=0}^{\infty} \frac{(-2 \Upsilon)^{\mathrm{n}}}{\mathrm{n} !} \frac{(-\beta)^{\mathrm{m}}}{2^{\mathrm{m}} \mathrm{m} !} \Gamma\left(\frac{\alpha+3+\mathrm{n}+2 \mathrm{~m}}{\alpha}\right)
\end{aligned}
$$

Also:

Where:

$$
\begin{aligned}
& \left(\mathrm{M}_{2}\right)^{\frac{3}{2}}=\left[\mathrm{E}\left(\mathrm{x}^{2}\right)\right]^{\frac{3}{2}}=[2 \Upsilon \mathrm{YK}(2, \Upsilon, \beta, \alpha)+ \\
& \beta \mathrm{K}(3, \Upsilon, \beta, \alpha)+\alpha \mathrm{K}(\alpha+1, \Upsilon, \beta, \alpha)]^{\frac{3}{2}}
\end{aligned}
$$

$$
\begin{aligned}
& \mathrm{K}(\alpha+1, \Upsilon, \beta, \alpha)=\frac{1}{\alpha} \sum_{\mathrm{n}=0}^{\infty} \sum_{\mathrm{m}=0}^{\infty} \frac{(-2 \Upsilon)^{\mathrm{n}}}{\mathrm{n} !} \\
& \frac{(-\beta)^{\mathrm{m}}}{2^{\mathrm{m}} \mathrm{m} !} \Gamma\left(\frac{\alpha+2+\mathrm{n}+2 \mathrm{~m}}{\alpha}\right)
\end{aligned}
$$

Kurtosis: The skewness of new mixture distribution denoted by:

$$
\text { C.K }=\frac{\mathrm{M}_{4}}{\left(\mathrm{M}_{2}\right)^{2}}-3
$$

where, $\mathrm{M}_{4}=\mathrm{E}\left(\mathrm{x}^{4}\right)=2 \Upsilon \mathrm{K}(4, \Upsilon, \beta, \alpha)+\beta \mathrm{K}(5, \Upsilon, \beta$, $\alpha)+\beta \mathrm{K}(\alpha+3, \Upsilon, \beta, \alpha)$

$\left[\mathrm{M}_{2}\right]^{2}=\left[\mathrm{E}\left(\mathrm{x}^{2}\right)\right]^{2}=\left[\mathrm{E}\left(\mathrm{x}^{2}\right)\right]^{2}=$

$2 \Upsilon \mathrm{K}(2, \Upsilon, \beta, \alpha)+\beta \mathrm{K}(3, \Upsilon, \beta, \alpha)+\alpha \mathrm{K}(\alpha+1, \Upsilon, \beta, \alpha)]^{2}$.

\section{Characteristic function}

Theorem 4: The Characteristic function of new mixture distribution is:

$$
\begin{aligned}
& \varphi_{\mathrm{x}}(\mathrm{it})=\mathrm{E}\left(\mathrm{e}^{\mathrm{itx}}\right)=\int_{0}^{\infty} \mathrm{e}^{\mathrm{itx}} \mathrm{f}(\mathrm{x}) \mathrm{dx}= \\
& \int_{0}^{\infty} \mathrm{e}^{\mathrm{itx}}\left(2 \Upsilon+\beta \mathrm{x}+\alpha \mathrm{x}^{\alpha-1}\right) \mathrm{e}^{-\left(2 \Upsilon \mathrm{x}+\frac{\beta}{2} \mathrm{x} 2+\mathrm{x}^{\alpha}\right)} \mathrm{dx}= \\
& \int_{0}^{\infty}\left(2 \Upsilon+\beta \mathrm{x}+\alpha \mathrm{x}^{\alpha-1}\right) \mathrm{e}^{-\left[(2 \Upsilon-i t) \mathrm{x}+\frac{\beta}{2} \mathrm{x}^{2}+\mathrm{z}^{\alpha}\right]} \mathrm{dx} \\
& \text { let } \mathrm{K}(\mathrm{t}, 2 \Upsilon-\mathrm{it}, \beta, \alpha)= \\
& \int_{0}^{\infty} \mathrm{e}^{-\left[(2 \Upsilon-\mathrm{it}) \mathrm{x}+\frac{\beta}{2} \mathrm{x}^{2}+\mathrm{x}^{\alpha}\right]} \mathrm{dx} \\
& \mathrm{K}(\mathrm{t}, 2 \Upsilon-\mathrm{it}, \beta, \alpha)= \\
& \frac{1}{\alpha} \sum_{\mathrm{n}=0}^{\infty} \sum_{\mathrm{m}=0}^{\infty} \frac{(2 \Upsilon-\mathrm{it})^{\mathrm{n}}}{\mathrm{n} !} \frac{(-\beta)^{\mathrm{m}}}{2^{\mathrm{m}} \mathrm{m} !} \Gamma\left(\frac{\mathrm{t}+\mathrm{n}+2 \mathrm{~m}+1}{\alpha}\right) \\
& \varphi_{\mathrm{x}}(\mathrm{it})=2 \Upsilon \mathrm{K}(\mathrm{t}, 2 \mathrm{Y}-\mathrm{it}, \beta, \alpha)+ \\
& \beta \mathrm{K}(\mathrm{t}+1,2 \mathrm{Y}-\mathrm{it}, \beta, \alpha)+\alpha \mathrm{K}(\mathrm{t}+\alpha-1,2 \mathrm{Y}-\mathrm{it}, \beta, \alpha
\end{aligned}
$$

Where:

$$
\begin{aligned}
& \mathrm{K}(\mathrm{t}+1,2 \Upsilon-\mathrm{it}, \beta, \alpha)= \\
& \frac{1}{\alpha} \sum_{\mathrm{n}=0}^{\infty} \sum_{\mathrm{m}=0}^{\infty} \frac{(2 \Upsilon-\mathrm{it})^{\mathrm{n}}}{\mathrm{n} !} \frac{(-\beta)^{\mathrm{m}}}{2^{\mathrm{m}} \mathrm{m} !} \Gamma\left(\frac{\mathrm{t}+\mathrm{n}+2 \mathrm{~m}+1}{\alpha}\right)
\end{aligned}
$$

And:

$$
\begin{aligned}
& \mathrm{K}(\mathrm{t}+\alpha-1,2 \Upsilon-\mathrm{it}, \beta, \alpha)= \\
& \frac{1}{\alpha} \sum_{\mathrm{n}=0}^{\infty} \sum_{\mathrm{m}=0}^{\infty} \frac{(2 \Upsilon-\mathrm{it})^{\mathrm{n}}}{\mathrm{n} !} \frac{(-\beta)^{\mathrm{m}}}{2^{\mathrm{m}} \mathrm{m} !} \Gamma\left(\frac{\mathrm{t}+\alpha+\mathrm{n}+2 \mathrm{~m}}{\alpha}\right)
\end{aligned}
$$

\section{CONCLUSION}

This study, propose a new mixture distribution, it is called "The New Mixture Distribution" which is expand the Weibull distribution in the analysis of data with real support.

This research include these results: mixing between the exponential and Rayliegh contain one parameter depending on the tail of each of them. Mixing between the exponential and Weibull contain one parameter in the same former style. Using the result from above to represent the new distribution called "New Mixture Distribution". Explaining the shapes of the density function and hazard rate function. Our realization of $\mathrm{r}^{\text {th }}$-moment, incomplete moment, the moment generating function and characteristic function. Finally, obtaining the quantile function and any other statistical properties of the new distribution. Hope that this research contributes to the development of the search for new distributions that accompany the process of economic and health development and benefit from the problems of survival and reliability analyzes. 


\section{APPENDIX}

This research using a special function called wright generalized hypergeometric function:

$$
{ }_{1} \psi_{0}\left[\begin{array}{c}
\left(\alpha_{1}, F_{1}\right), \ldots,\left(\alpha_{s}, F_{s}\right) \\
\left(\beta_{1}, D_{1}\right), \ldots,\left(\beta_{j}, D_{j}\right)
\end{array} ; x\right]=\sum_{n=0}^{\infty} \frac{\prod_{m=0}^{s} \Gamma\left(\alpha_{m}+F_{m} n\right) x^{n}}{\prod_{m=0}^{j} \Gamma\left(\beta_{m}+D_{m} n\right) n !}
$$

The meijer function is:

$$
\begin{aligned}
& \int_{0}^{\alpha} x^{\alpha-1}(a-x)^{\beta-1} G_{s j}^{m n}\left(\left.\omega x^{\frac{1}{c}}\right|_{s} ^{a_{s}}\right) d x \\
& =\frac{c^{\mu} 1^{-\beta} \Gamma(\beta)}{(2 \pi)^{w^{*}(c-1)} j^{1-(\alpha-\beta)}} G_{c s+1 c j+1}^{c m, n+1} \\
& \left(\frac{\omega^{c} j^{1}}{c^{c}(j-s)} \Delta(1,1-\alpha), \Delta\left(c, b_{j}\right), \Delta(1,1-\alpha-\beta)\right.
\end{aligned}
$$

Where:

$$
\mu=\left(\sum_{\mathrm{k}=1}^{\mathrm{j}} \mathrm{b}_{\mathrm{k}}-\sum_{\mathrm{k}=1}^{\mathrm{s}} \mathrm{a}_{\mathrm{k}}+\frac{\mathrm{s}-\mathrm{j}}{2}\right)+1
$$

And $\mathrm{a}_{\mathrm{k}}-\mathrm{b}_{\mathrm{k}} \neq 1,2, \ldots,\left(\mathrm{a}_{\mathrm{k}}, \mathrm{b}_{\mathrm{k}}\right.$ and $\left.\alpha\right)$ are complex parameters and (c and 1$)$ are co-prime numbers.

$$
\mathrm{Pw}^{*}=\mathrm{m}+\mathrm{n}+\frac{\mathrm{s}+\mathrm{j}}{2}
$$

\section{REFERENCES}

Abramowitz, M. and I.A. Stegun, 1970. Handbook of Mathematical Functions. Dover, New York.

Cordeiro, G.M. and M. de Castro, 2011. A new family of generalized distributions. J. Stat. Comput. Simul., 81: 883-898.
Cordeiro, G.M., E.M.M. Ortega and A.J. Lemonte, 2014. The exponential-weibull lifetime distribution. J. Stat. Comput. Simul., 84: 2592-2606.

Jeffrey, A. and D. Zwillinger, 2007. Table of Integrals, Series and Products. 7th Edn., Academic Press, New York, ISBN-13:978-0-12-373637-6, Pages: 1170.

Kiryakova, V.S., 2002. Meijers g-function: Bulgarian traces for its use in special functions, integral transforms and fractional calculus. Proceedings of the 31 st Spring Conference on Union of Bulgarian Mathematicians, April 3-6, 2002, Borovets, Bulgaria, pp: 25-35.

Merovci, F. and I. Elbatal, 2015a. A new generalization of liner exponential distribution theory and application. J. Stat. Appl. Probab. Lett., 2: 1-14.

Merovci, F. and I. Elbatal, 2015b. Weibull rayleigh distribution: Theory and applications. Intl. J. Appl. Math. Inf. Sci., 9: 2127-2137.

Nadarajah, S. and S. Kotz, 2005. On some recent modifications of Weibull distribution. IEEE. Trans. Reliab., 54: 561-562.

Nasiru, S., 2016. Serial weibull rayleigh distribution: Theory and application. Intl. J. Comput. Sci. Math., 7: 239-244.

Prudnikov, A.P., Y.A. Brychkov and O.I. Marichev, 1986. Integrals and Series: More Special Functions. 3rd Edn., Gordon \& Breach Science Publishers, Philadelphia, Pennsylvania, USA., ISBN: 9782881246821, Pages: 800.

Wright, E.M., 1935. The asymptotic expansion of the generalized hypergeometric function. J. London Math. Soc., 10: 286-293.

Xie, M. and C.D. Lai, 1996. Reliability analysis using an additive Weibull model with bathtub-shaped failure rate function. Reliab. Eng. Syst. Safety, 52: 8793. 